\title{
Malaria burden through routing reporting: Relationship between incidence estimates
}

\author{
Simon P. Kigozi ${ }^{1,2}$ \\ ${ }^{1}$ Disease Control, London School of Hygiene \& Tropical Medicine, Kampala, Uganda, ${ }^{2}$ Infectious disease Research Collaboration, Kampala, \\ Uganda
}

Objective

To evaluate the relationship between test positivity rate and test-confirmed malaria case rate both in time and space, to provide better understanding of the utility and representativeness of HMIS data for changing malaria burden in endemic settings.

\section{Introduction}

Routine surveillance is an important global strategy for malaria control. However, there have been few studies comparing routine indicators of burden, including test positivity rate (TPR) and test-confirmed malaria case rates (CMCR), over spatial and temporal scales.

\section{Methods}

We studied the relationship between these indicators in children under 11 years, presenting with suspected malaria at outpatient department clinics in three health facilities in locations of varying transmission across Uganda. We evaluated trends in each indicator over time (month) and space (by village) and explored the relationship between them using multivariable regression models.

\section{Results}

Overall, 65,710 participants visited the three clinics during the study period. Pairwise relationships between TPR and CMCR showed good agreement over time, particularly for TPR's below 50\% and during low transmission seasons, but the relationship was complex at the village level. Village mean annual TPR remained constant while CMCR was drastically reduced with increased distance from health facility, highlighting health care access' importance. The forth quartile of distance from the health facility, relative to the first, was associated with reduction in CMCR with IRR of 0.40 (95\% CI: $0.23-0.63$; $\mathrm{p}=0.001), 0.55(0.40-0.75 ; \mathrm{p}<0.001)$, and $0.25(0.12-0.51 ; \mathrm{p}<0.001)$ for Nagongera, Kihihi, and Walukuba respectively. Regression analysis results emphasized a non-linear (cubic) relationship between TPR and CMCR, after accounting for month, village, season and demographic factors.

\section{Conclusions}

Altogether, these results suggested a strong non-linear relationship between the two indicators regardless of transmission setting. However, the current malaria surveillance system in Uganda may under-represent burden from patients living furthest from sentinel health facilities.

\section{Acknowledgement}

We would like to thank all the: staff at the three health centre IV's of Nagongera, Walukuba, and Kihihi; the Uganda malaria surveillance project data officers at these sites; and, the wider UMSP/IDRC team that have been instrumental to the smooth running of this surveillance work over the years. 